\title{
Dielectric breakdown in a simplified parallel model
}

\author{
Howland A. Fowler, ${ }^{\text {a) }}$ Judith E. Devaney, ${ }^{\text {b) }}$ and John G. Hagedorn ${ }^{\text {c) }}$ \\ High Performance Systems and Services Division, Information Technology Laboratory, National \\ Institute of Standards and Technology (NIST), Gaithersburg, Maryland 20899 \\ Francis E. Sullivan ${ }^{\mathrm{d})}$ \\ Center for Computing Sciences, Institute for Defense Analyses, Bowie, Maryland 20715
}

(Received 5 February 1998; accepted 11 June 1998)

\begin{abstract}
The growth of streamer trees in insulating fluids (a submicrosecond process that triggers high-voltage breakdown) has been simulated with a combination of parallel-coding tools. Large grids and arrays display well the multifractal, self-avoiding character of the streamer trees. Three physical cases have been approximated by different power-law weightings of the statistical growth filter: dense anode trees, in the uniform field; sparse cathode trees (a rarer experimental case); and ultrasparse anode trees (seen in some fluids of higher viscosity). The model is contained in a software package that is written in Fortran 90 with data parallel extensions for distributed execution. These extensions encapsulate an underlying, invisible message-passing environment, thus enabling the solution of memory-intensive problems on a group of limited-memory processors. Block partitioning creates processes of reasonable size, which operate in parallel like small copies of the original code. The user needs only to express his model in transparent array-directed commands; parallel interfacing between blocks is handled invisibly. Breakdown is performed in parallel in each of the local blocks. Results are presented for experiments run on eight and nine nodes of the IBM SP2, and four and eight nodes of the SGI Onyx and Origin, three examples of multiple-processor machines. Display is carried out in three dimensions. Timing of the growth can be shown by color banding or by frame animation of the results. The adequacy of the growth rules and size scaling are tested by comparing the simulations against snapshots from high-voltage discharge events. [S0894-1866(98)01805-7]
\end{abstract}

\section{INTRODUCTION}

The growth of fast streamer trees in liquid-dielectric insulation provides the precursor "leader' conduction path through which damaging flashover between electrodes can take place. The high-speed, variable nature of this phenomenon has made its detailed mechanism elusive. Nonetheless, a global description of the process may be useful for its characterization.

We have applied stochastic Laplacian growth as a model for fast streamer trees in liquid dielectrics. Filamentary dielectric breakdown has been extensively discussed by Pietronero and Wiesmann ${ }^{1}$ and by others. ${ }^{2-5}$ Here we construct a practical realization of the algorithm on a large Cartesian grid using boundary conditions that confine the electric field. We examine the effect of parameters (threshold voltage, choice of power law) on the fractal structure and timing of the growth process.

The calculation of the voltage field throughout the full volume, repeated after each stage of breakdown growth, is the major computational burden that calls for parallel methods.

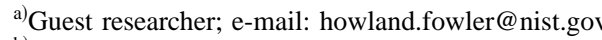

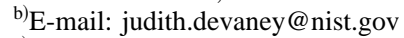

${ }^{c}$ E-mail: john.hagedorn@nist.gov

${ }^{d)}$ E-mail: fran@super.org
}

The single-instruction-stream, multiple-data (SIMD) model fits our problem closely. We had tested an earlier machine-language version of our algorithm on the CM-2 Connection Machine. ${ }^{6}$ The SIMD version there was synchronous and specific to the machine. The present method uses block partitioning under Message Passing Interface (MPI) control, ${ }^{7}$ in a single-program, multiple-data (SPMD) approach, which runs asynchronously. For a data-parallel environment that is easy to use and transport, we have written the code in array-directed commands of Fortran 90, together with our library DPARLIB ${ }^{8,9}$ that encapsulates the underlying MPI calls. ${ }^{7}$ This will run in any environment that is Fortran 90 and MPI enabled, be it networks of workstations, symmetric multiprocessors (SMPs), parallel machines, or some combination of these.

Running Fortran 90 on a single processor does not provide access to the memory that large data-parallel programs require. Our software has been designed not only to fit into the Fortran 90 syntax transparently, but also to run across the nodes of a group multiprocessor. It extends the array-directed commands seamlessly, across block boundaries. For development purposes, the number of processors executing can be reduced to one, like a serial version of the code using only plain Fortran 90 commands. These features enable users to test small versions of their code on their workstations. Then, with only minor changes in the code, much larger versions can be run on machines like the SP-2, 


\section{PHYSICAL MODEL AND ALGORITHM}

The elements of a stochastic fractal model have been demonstrated in numerous examples. ${ }^{10-16}$ The streamer tree is assumed to be fully conductive and electrically attached to the electrode from which it originates. The voltage field is defined over a set of points in a rectangular grid. The streamer tree is a connected set of those points.

After initialization, the algorithm alternates between a Laplacian convergence procedure and statistically weighted streamer tree growth. Our algorithm may be summarized as follows:

Initialize voltage field and anode/cathode configuration Set the streamer tree to be the anode (including starter needle)

Run Gauss-Seidel over-relaxation on Laplace's equation for 200 iterations

Do until streamer tree has reached cathode

Set all sites on streamer tree to voltage 0.0

Set all sites on cathode to voltage 1.0

Solve Laplace's equation via Gauss-Seidel over-relaxation

Find nearest neighbors to tree

Do until the set of breakdown sites is not less than one Set Breakdown sites equal to the empty set Choose, locally in each block, red or black (at random)

Set breakdown candidates equal to neighbors of the color

Remove candidates with voltage below a specified threshold

For each remaining site generate a weighted random number

Remove breakdown sites whose voltage is

End Do below this number

Add breakdown sites to streamer tree

\section{End Do}

The two stages of this algorithm are the solution of Laplace's equation and the growth of the streamer tree. We now consider these in more detail.

\section{A. Solution of Laplace's equation}

Laplace's equation is fully solved throughout the interior region (filled with dielectric liquid) using the anode, starter needle, and streamer tree as one boundary at zero potential and the plane cathode as the counterelectrode. Sides of the cube-volume support periodic boundary conditions. The quasistatic field approximation (i.e., that the voltage field always "catches up" with the new boundary condition at each stage of the growth) is physically reasonable, since breakdown streamers are known to advance at supersonic velocities, still slow by comparison with the speed of light in the dielectric liquid.

A first-order, six-point-averaging Laplacian operator ${ }^{17}$ vances through Gauss-Seidel overrelaxation. Initially 200 iterations of convergence bring the smoothing precision below one part in 10,000 , on a grid of $128 \times 128 \times 128$. (Nominal single iteration time is roughly $10 \mathrm{~s}$.) After this first high-precision convergence, the epsilon is relaxed (to 0.001-0.005) in the interests of speed. Because each growth stage creates only small perturbations on the existing boundary conditions, the later cycles reconverge rapidly, in 5-10 iterations. The changes propagate in rapidly diminishing ripples, away from the newest growth links on the tree.

\section{B. Growth of the streamer tree}

Grid sites immediately adjacent to the tree are examined. If their voltages exceed a specified threshold (or cutoff) level, then the surviving voltages are compared against a weighted distribution of random numbers. Those that pass over the statistical hurdle are attached to the tree, and the Laplacian convergence is recycled for the new, perturbed boundary condition. Field and growth stages alternate until the counterelectrode is reached.

By contrast with some of the stochastic fractal models cited earlier, which recalculate Laplace's equation after each single new-growth site is added, our algorithm considers simultaneous, distributed growth possibilities on all "red" or all "black" neighbors of the tree during each growth stage. Growth is favored near the tips of the tree, where electrostatic field lines converge strongly and the voltage gradients are largest. While the details of the mechanism for fast streamer growths are still to be determined from experiment, we ask, "To what laws of shape and timing does the observed growth conform?"

Weighting of the growth-probability power law, as a function of electric field strength, determines the degree of bushiness (or fractal dimension) of the final structure. At one extreme, Sanchez et al. ${ }^{18}$ have shown that a fourthpower (or higher) dependence on field strength produces a single, self-avoiding strand that resembles a directed random walk. Side branching is minimized. At the other limit, Witten and Sander ${ }^{19}$ showed that a linear filtering against uniformly distributed random numbers results in a dense growth form, which is also found in diffusion-limited aggregation, from a different mechanism. In this linear regime, a mass of twigs advances with a "front" of thickly spaced growing tips. A recent review by Erzan, Pietronero, and Vespignani ${ }^{20}$ considers ways of characterizing fractal dimension in this class of problems.

In Fig. 1 we see a log-log plot of breakdown probability (for a single link between grid vertices) versus power of the electric field strength, measured as the voltage difference between the conducting tree, at zero potential, and its immediate neighbors on the Cartesian grid. The power law determines the likelihood of growth from the tip, as against side branching. Recall that Laplace's equation is size-scalable without limit. By adopting a power-law probability, we may expect self-similar growth over large ranges of both size and field strength. This is not viewed as a microscopic description, but rather as a law controlling the global growth form.

Notice how minuscule the breakdown probability becomes for cube-law and higher powers on the left half of 
WEIGHTED BREAKDOWN LAWS

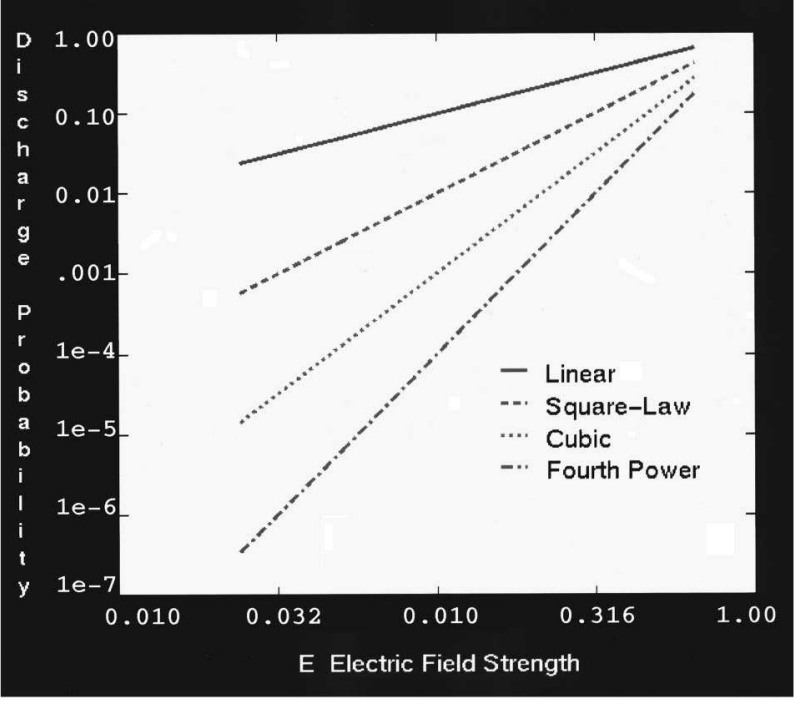

Figure 1. Log-log plot of breakdown probability (discharge probability for an individual link) versus electric field strength for power laws with integral exponents. The horizontal scale is electric field strength at the candidate links in the discretized step approximation (difference between the zero voltage of the streamer tree and the voltages at the neighbor grid nodes). The limit value 1.0, to which all fields are normalized, is the value that would be reached between flat-plate electrodes separated by one grid step.

the graph. The range shown, of field strengths, is that encountered in growth across a $128 \times 128 \times 128$ grid volume. As the streamer tip advances from anode to cathode, the diminishing gap leads to progressively higher fields around the leading branches, while electrostatic screening causes the fields around the base of the tree to drop back below threshold, so that growth is cut off there. Because the electric field is confined by limiting boundary conditions, and the growth process starts and stops at an abrupt threshold, we do not expect a "pure" fractal dimension throughout the growth. The tree is sparser as it leaves the concentrating needle on the primary electrode, and denser as it approaches the counterelectrode.

The algorithm must operate uniformly across a wide range of growth rates. For square-law and higher powers, the starting probability near the anode needle (where the field strength only slightly exceeds the threshold value of $0.015-0.10$ ) is very small-tens or hundreds of empty statistical tries may occur between single breakdown events. By contrast, linear weighting leads to tens of events per growth stage, in the shorter gaps, approaching the cathode.

The uniform treatment of slow and fast stochastic processes assumes that the discretized physical model approximates a Markov-process master equation, ${ }^{21,22}$ whose time variable is proportional to the number of trial instances.

\section{IMPLEMENTATION}

Our code was first implemented in a serial version, on Sun and SGI workstations, and was run interactively on small detailed printouts to the screen. This serial code was implemented in standard Fortran 90 and exploits that language's array-oriented syntax and intrinsic functions. As examples of the powerful simplifications available, we describe some of the details.

The code makes extensive use of array arithmetic, the WHERE construct, array functions such as CSHIFT, MAXVAL, COUNT, and the dynamic-memory-allocation features of Fortran 90. Our program logic is expressed through combinations of real and logical arrays, the WHERE assignment mask of Fortran 90, and a selection of spreading and collecting operations.

For example, our Laplacian-operator subroutine makes vigorous use of Fortran 90's CSHIFT operation. The sum of neighbors calculation was implemented in the serial version with the following code:

$$
\begin{aligned}
\text { blksum }= & \text { cshift }(\text { blkphi, }+1,1)+\text { cshift }(\text { blkphi, }-1,1) \& \\
& + \text { cshift }(\text { blkphi, }+1,2)+\text { cshift }(\text { blkphi, }-1,2) \& \\
& + \text { cshift }(\text { blkphi, }+1,3)+\text { cshift }(\text { blkphi, }-1,3)
\end{aligned}
$$

CSHIFT is the standard Fortran 90 intrinsic function that performs circular shifts. The array (blksum) that results from this calculation contains at each grid point the sum of the six neighbors of that grid point in the original array, using periodic boundary conditions. The division of the three-dimensional grid into alternating/adjacent red and black subgrids is a feature brought over from statisticalmechanics simulations. Black-to-red and red-to-black averaging steps are carried out across all processes, at each loop. This routine is global over the entire internal voltagefield array.

We also made extensive use of the "modules" feature of Fortran 90. This simplified the debugging process by enabling the compiler to catch certain types of coding errors. In particular, the compiler checked that all subroutine calls had properly constructed calling sequences.

The statistical selection was coded by the following steps. By contrast with the Laplacian routine, these are local operations, executed independently in each block.

(1) Find all nearest neighbor sites to the growth tree within the process block. This procedure produces a logical mask array that is .true. at the neighbor sites and .false. elsewhere.

(2) Select, at random for each block, either the red or black neighbors within that block. The mask array of neighbors is modified to reflect this choice. Since we are considering simultaneous but independent growth across the full distributed array of neighbor sites, this $\mathrm{red} / \mathrm{black}$ choice discourages selection of adjacent sites, which might lead to growth concentration or cause a locally enhanced perturbation of the voltage field.

(3) Allocate a one-dimensional REAL array with one element for each surviving neighbor. If a power law higher than linear is desired, then additional arrays of the same length are allocated.

(4) Fill the one-dimensional array(s) with uniformly distributed random numbers. The DPARLIB dp_uni is used to generate random numbers that are independent across all of the parallel processes. 
(5) Calculate the MAX array of these random arrays. The resulting array contains a weighted distribution at one power lower than the desired breakdown power law. Step (7) below, the statistical filtering, is equivalent to a single order of integration for the distribution of voltages that will survive this hurdle.

(6) UNPACK the MAX array through the neighbor mask array. This creates a three-dimensional array with the weighted random numbers at the surviving neighbor sites.

(7) Compare the voltages to the weighted random array at the neighbor sites. WHERE they pass, add new sites to the existing growth tree. Write out their grid positions, trial numbers, and field strengths.

(8) If no sites have been added to the tree, return to step (2).

(9) If a site has been added to the tree, return to the Laplacian-convergence stage.

Note that the approach used in steps (3)-(6) substantially reduces memory and computational requirements by generating the weighted distribution of random numbers in minimally sized arrays. The resulting linear array is UNPACKed into the full-size three-dimensional array only when necessary.

The Fortran 90 array instructions that we needed were WHERE, CSHIFT, UNPACK, MAXVAL, MAX, MOD, COUNT, ANY, ALLOCATE, DEALLOCATE. In the blockparallel mode, some are used in local fashion by the individual processor, addressing only the portions of the major arrays seen within its own block.

\section{CONVERSION TO PARALLEL OPERATION}

The DPARLIB operations, which were required as substitutions or additions to enable parallel operation, were dp_cshift, dp_uni, dp_count, dp_any, dp_sync. ${ }^{8,9}$ In addition, the $\mathrm{dp}$ _initialize and dp_query_layout routines were called at the start of the run to set up the division of domains between processors.

The adaptation of the serial code was accomplished through the use of the DPARLIB subroutine library. This is a Fortran 90 library developed at NIST to facilitate the transition of serial applications to a parallel-computing environment and the development of new parallel programs. DPARLIB is built on MPI, an industry-standard library for passing data and coordinating the activities of multiple processes in a parallel operating environment.

DPARLIB is designed to be used in the SPMD programming approach. In other words, multiple copies of the same program are running simultaneously, and each copy is processing a different portion of the data. In particular, DPARLIB provides simple mechanisms to divide very large arrays into blocks, each of which is handled by a separate copy of the program. In practice, this means that the researcher can write parallel code that looks almost identical to serial code. In our case, the code could be written as though addressed to a single active grid node and its immediate neighbors. Fortran 90, extended across block boundaries by DPARLIB, executed each instruction on all $2,000,000$ sites of each array.

In this way, an existing serial program can often be converted to a parallel program with few changes by using block parallelism.

DPARLIB's emphasis on array handling is designed to mesh with Fortran 90's array syntax and intrinsic arrayhandling functions. Much of DPARLIB consists of parallel versions of the intrinsic array functions such as CSHIFT and MAXVAL. Because DPARLIB is coded entirely in standard Fortran 90 and depends only on MPI, it is portable to any environment that provides those two resources.

The adaptation of the dielectric breakdown code was straightforward and involved the following steps.

(1) Add calls to DPARLIB housekeeping functions for initialization and specification of the mapping of data arrays to an array of processes. (dp_initialize and dp_query_layout).

(2) Make all distributed data arrays allocatable, and allocate the arrays based on information provided by DPARLIB calls.

(3) Change various Fortran 90 intrinsic calls to the corresponding DPARLIB calls. This simply involves changing the name of the called routine, e.g., cshift becomes dp_cshift and maxval becomes dp_maxval.

Once this conversion was complete, each process ran the same program with its own block of data. It used no explicit reference to data within other blocks, except as information was received through collecting, spreading, and shifting operations in DPARLIB. The parallel version could be run at once, on a network of workstations with the local area multicomputer (LAM) implementation of MPI ${ }^{23}$

Our Laplacian operator is first-order and requires only the exchange of data for single layers between domain blocks. $^{24}$

A convenient feature of DPARLIB is that communication between processes is handled automatically. Thus, the typical boundary-layer transfers of data, described by Gropp, Lusk, and Skjellum, ${ }^{7}$ do not require explicit consideration by the code writer. dp_cshift includes-invisiblythe necessary "shadow" sites and cross-boundary transfers of data that must take place at the planes between process domains. ${ }^{17,24}$ The user can ignore this complex and errorprone aspect of code writing.

There were, however, two crucial points in this program at which we had to take some care about the parallel execution of multiple copies of the code: writing the streamer tree sites to a disk file and testing for whether the streamer tree had reached the cathode. At these points, the application code must explicitly deal with the fact that multiple copies are executing in parallel.

At the end of the tree-growth part of the algorithm, the grid sites that have been added to the streamer tree are written to a data file. Because these sites will be found in multiple processes, we have these multiple processes trying to write to the same output file at the same time. This will result in errors on many systems, including the Sun, SGI, and IBM systems on which we were running these tests.

This problem can be solved by having each process write to its own output file. These separate output files could then be merged after the program is complete. Where multiprocessor access to a common file is possible, we instead implemented a "round-robin" solution that involves the synchronization of the processes using the DPARLIB rou- 


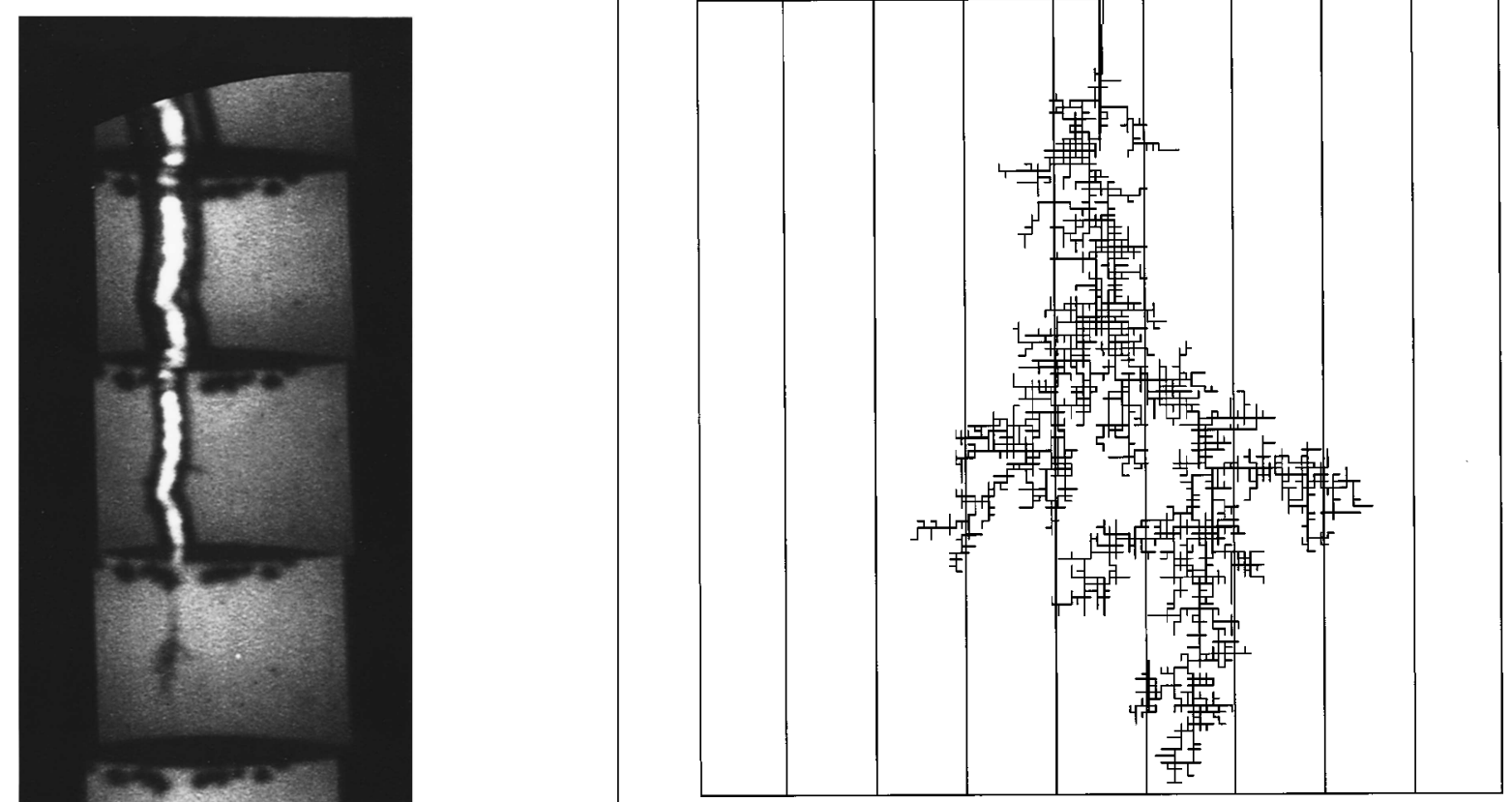

Figure 7. Lateral projection of a square-law-weighted streamer simulation on a grid of $126 \times 126 \times 126.4227$ statistical tries produced 4432 discharged links. The threshold voltage was set at 0.015. To compensate for the very low starting probability, all voltage values on neighbor sites were raised by 0.020 when comparing against the statistical filter. Real time was 13:32:37.

tical test, after the manner of the algorithm of Bortz, Kalos, and Lebowitz ${ }^{25}$ so that the a priori likelihood of at least one discharge event is increased to one.

The changed routine for higher powers proceeds as follows, after step (3) in the sequence that was described earlier in Sec. II.

Figure 6. Fast (secondary) cathode streamer, produced under conditions similar to those in Fig. 3. The cathode is at the top, the anode at the bottom. The time sequence now proceeds from bottom to top. The tree structure is noticeably sparser. This event occurs with distinctly lower probability than the fast anode streamer. The bushy growth attached to the cathode at the top is the slower (primary) streamer. Both anode and cathode fast streamers provide the leader breakdown path that leads to high current flow and the formation of the shock wave.

"leader'" conduction path from needle to counterelectrode, shown in heavy line weight, resembles a directed random walk. In some instances, two branches of the leader may reach the counterelectrode on the same statistical try.

\section{TIME COMPRESSION FOR HIGHER POWERS}

The extremely low probabilities shown in the lower half of Fig. 1 complicate the trial of cube-law and higher powers. For low values of initial field strength, many thousands of empty statistical trials would be required to secure a very few steps of growth. Our solution is to reweight the statis-

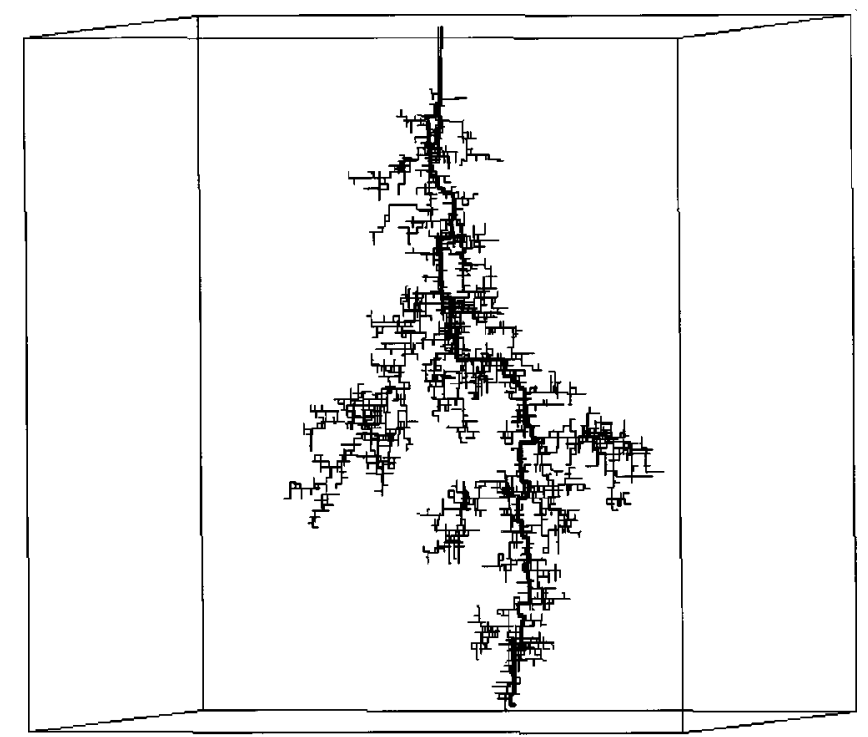

Figure 8. Isometric view of Fig. 7. Compare against Fig. 6. The cathode is now at the top, the anode at the bottom. 


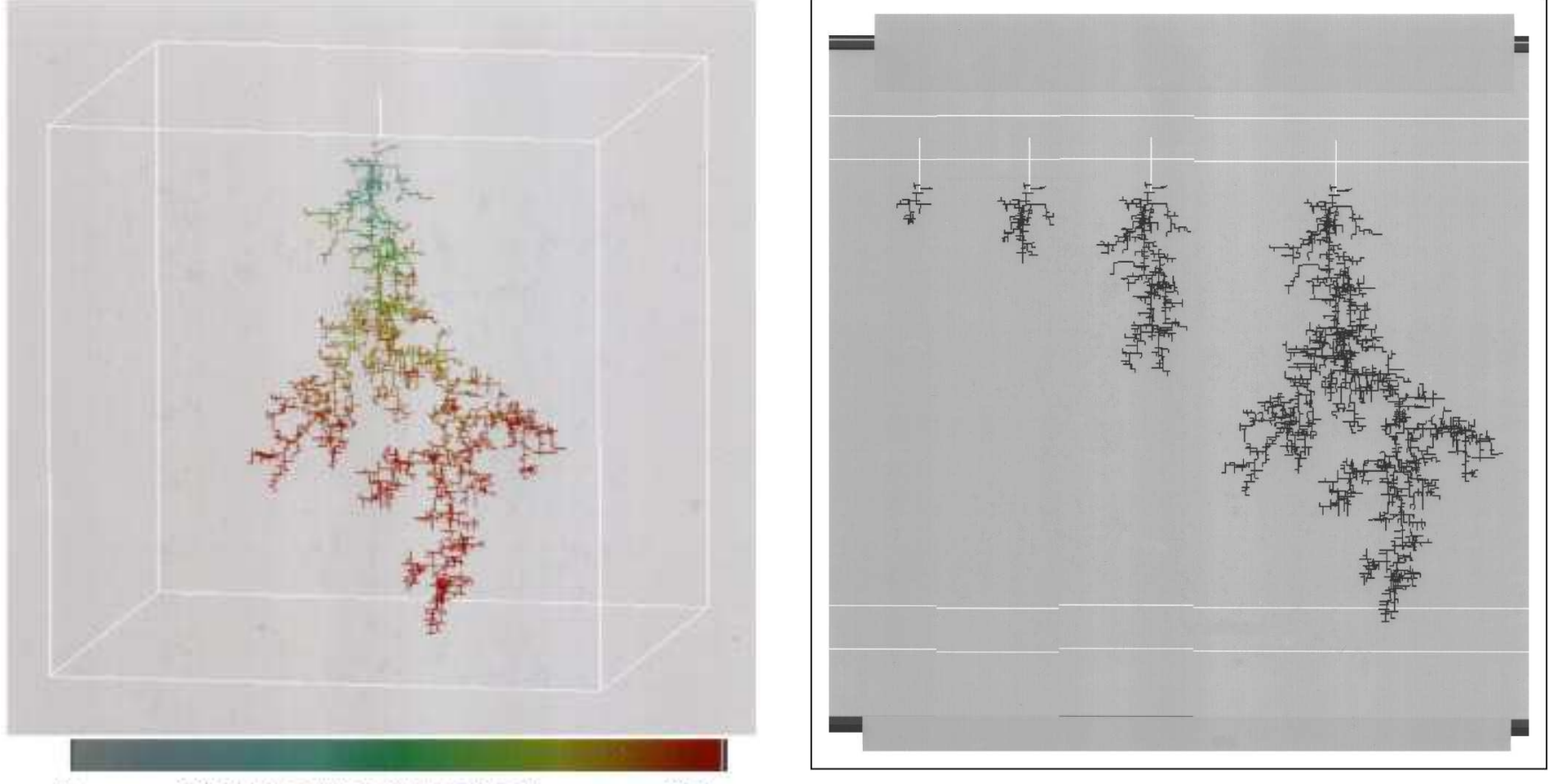

\section{0 \\ Trial Number (Monte Carlo Time) \\ 4227}

Figure 9. Color-banded version of the streamer tree shown in Fig. 8. The color palette is a linear representation of cumulative statistical tries, a measure of Monte Carlo time.

(a) PACK the surviving neighbor-voltage values onto the first linear real array.

(b) square, cube, or otherwise exponentiate this array onto a second linear array. Sum the values of the elements in this array to a value we call "sumsq." It represents the a priori probability of at least one discharge event.

(c) Fill a third linear array, of the same length, with uniformly random numbers. Multiply these by the coefficient "sumsq" - this is equivalent to "compressing" Monte Carlo time. It is also equivalent to the normalization used in many previous examples of algorithms where only the fractal shape, and not the timing, were sought.

(d) WHERE the second array fails to exceed the random array, weighted by the coefficient "sumsq," set the values of the first linear array equal to zero.

(e) UNPACK the revised first array through the position mask of the original neighbors.

(f) WHERE the unpacked 3D array is now greater than zero, add new sites to the existing tree.

(g) Update the statistical trial number (Monte Carlo time tick) by an integer equal to $\operatorname{FLOOR}((\log (\mathrm{rho}) /$ $\log ($ sumsq) $)+1)$, where rho is a random number. ${ }^{25}$ This estimates rather than counts the number of empty trials before a successful trial in which growth is added.

(h) Return to the Laplacian-convergence subroutine.

Again, Fortran 90 has furnished powerful arraydirected operations for composing the program logic. The
Figure 10. Animated screen development of Fig. 8 against cumulative statistical tries (Monte Carlo time). The acceleration of growth across the second half of the gap is worth noting. Note that the duration of the full growth would correspond to a period of around $30 \mathrm{~ns}$ or less than the interval between frames in Figs. 3 and 6.

additional computing burden is small, since these operations are performed on few elements, numbering only in the thousands.

The modified algorithm has been successfully run for cube-law and fourth-power examples. Figure 11 illustrates simulation of a cube-law streamer, run on a $128 \times 128$ $\times 128$ grid, with a short anode needle, two grid steps in length. The spareness, restricted lateral branching, and directed alignment are characteristic of growth with this exponent in the power law.

Recent reports by Miyano and collaborators ${ }^{29}$ display fast anode streamers in D40 perfluoropolyether and FC10 perfluorocarbon. Photographs of them show strong similarities to Fig. 11.

Thus, we note that physical counterparts to our powerlaw simulations with exponents 1,2, and 3 are found in the experimental observations on fast filamentary streamers.

\section{LIMITATIONS}

There are several clear limits in the present realization of the model.

(1) Diagonal breakdown paths between grid nodes have not been included in the simulations shown here. Increased flexibility of directional choice, at each event, would be a step towards greater physical realism, albeit imposing a greater bookkeeping burden. (This capability is being added.)

(2) The Laplacian-convergence accuracy has been relaxed in a manner that may tend to favor tip-growth versus 
of computers and workstations. LAM is an open cluster environment for workstations or multiprocessors. ${ }^{23}$

Our dielectric-breakdown code, named CADMUS, will be available through our Web site http://www.itl.nist.gov/ div895/sasg/.

\section{ACKNOWLEDGMENTS}

The authors are indebted to many colleagues for their useful participation and help. The authors' first ideas for solving the problem came from the ultra-high-speed photographic experiments of Ed Kelley, Ken Stricklett, and collaborators in the NIST EEEL Applied Electrical Measurements group. Suggestions from Jacques Amar helped them understand Monte Carlo time, and Jack Douglas has contributed valuable insights about Laplacian fractals. Jim Sims rendered major assistance with workstations, Fortran programming, and graphics (the routine FRAMES). Tere Griffin contributed color and animation to the results. Howard Hung performed the porting and timing comparisons. Larry Davis and Mitchell Murphy, University of Maryland Institute for Advanced Computing Systems, helped them greatly with their earlier SIMD version of the algorithm, adapted for the CM-2 version of the connection machine. Charles Fenimore has been a constructive critic and collaborator. One of the authors (H.A.F.) expresses his warm thanks to the NIST Information Technology Laboratory for access and guest-researcher technical privileges granted during 1996-1997.

Certain commercial equipment and software may be identified in order to specify or describe adequately the subject matter of this work. In no case does such identification imply recommendation or endorsement by the $\mathrm{Na}-$ tional Institute of Standards and Technology, nor does it imply that the equipment or software is necessarily the best available for the purpose.

\section{REFERENGES}

1. L. Pietronero and H. J. Wiesmann, Z. Phys. B 70, 87 (1988).

2. W. G. Chadband, IEEE Trans. Electr. Insul. 23, 697 (1988).

3. W. G. Chadband and T. M. Sufian, IEEE Trans. Electr. Insul. 20, 239 (1985).

4. S. Satpathy, Fractals in Physics (Elsevier Science, Amsterdam, 1986), p. 173.

5. T. J. Lewis, in The Liquid State and its Electrical Properties, edited by E. E. Kunhardt, L. G. Christophorou, and L. E. Luessen, Proceedings the NATO Advanced Study Institute, 5-17 July 1989, Sintra, Portugal (Plenum, New York, 1988), pp. 431-453.

6. Larry Davis and Mitchell Murphy of the University of Maryland Institute for Advanced Computing Systems provided access to the CM-2. bridge, 1994), especially pp. 60-80

8. J. E. Devaney and J. Hagedorn, National Institute of Standards and Technology internal paper, May 7, 1996.

9. J. Hagedorn and A. Heckert, DPARLIB User's Guide, draft of NIST software library documentation, March 18, 1997.

10. L. Niemyer, L. Pietronero, and H. J. Wiesmann, Phys. Rev. Lett. 52, 1033 (1984)

11. L. Pietronero and H. J. Wiesmann, J. Stat. Phys. 36, 909 (1984).

12. H. J. Wiesmann and L. Pietronero, in Ref. 4, p. 151.

13. L. Pietronero, C. Evertsz, and H. J. Wiesmann, in Ref. 4, p. 159.

14. M. Murat, in Ref. 4, p. 169.

15. E. Arian, P. Alstrom, A. Aharony, and H. E. Stanley, Phys. Rev. Lett. 63, 2005 (1989).

16. P. D. Beale and P. M. Duxbury, Phys. Rev. B 37, 2785 (1988).

17. J. J. Modi, Parallel Algorithms and Matrix Computation (Clarendon, Oxford, 1988), pp. 135-143.

18. A. Sanchez, F. Guinea, L. M. Sander, V. Hakim, and E. Louis, Phys. Rev. E 48, 1296 (1993). In these treatments, idealizations such as radial growth to a remote spherical-shell boundary, with normalized probabilities, favor growth having a simple, well-defined fractal dimension.

19. T. A. Witten and L. M. Sander, Phys. Rev. Lett. 47, 1400 (1981); Phys. Rev. B 27, 5686 (1983).

20. A. Erzan, L. Pietronero, and A. Vespignani, Rev. Mod. Phys. 67, 545 (1995).

21. M. M. Clark, L. M. Ruff, and H. L. Scott, Comput. Phys. 10, 584 (1996).

22. K. Binder and D. W. Heermann, Monte Carlo Simulation in Statistical Physics (Springer, Berlin, 1988), especially, pp. 28-29.

23. D. Burns and R. B. Daoud, Supercomputing Symposium '94, June 1994, Toronto, Canada; code available at http//www.osc.edu/ lam.html.

24. For a similar treatment of a second-order operator, see, for example, A. Villareal and J. A. Scales, Comput. Phys. 11, 388 (1997), especially p. 392.

25. I. Beichl and F. E. Sullivan, Comput. Sci. Eng. 4, 91 (1997); see also A. B. Bortz, M. H. Kalos, and J. L. Lebowitz, J. Comput. Phys. 17, 10 (1975) especially Appendix B; J. G. Amar and F. Family, Phys. Rev. Lett. 74, 2066 (1995).

26. K. L. Stricklett, E. F. Kelley, H. Yamashita, H. Kawai, and C. Fenimore Conference Record, IEEE International Symposium on Electrical Insulation, 1990, pp. 161-164. These data were taken by means of a high-speed "optical storage line" technique which limited the available spatial resolution. Dr. Stricklett made his original data available to the authors.

27. P. K. Watson, Conference Record of the 10th International Conference on Conduction and Breakdown in Dielectric Liquids, Grenoble, France, 10-14 September 1990, pp. 22-28.

28. C. Fenimore, Conference Record, IEEE International Symposium on Electrical Insulation, Cambridge, MA, 5-8 June 1988, pp. 27-30.

29. K. Miyano, K. Yamazawa, M. Pompili, C. Mazzetti, and H. Yamashita, 1997 Annual Report of the Conference on Electrical Insulation and Dielectric Phenomena, Minneapolis, 19-22 October 1997, Vol. II, pp. 640-643 (available from IEEE Service Center, Piscataway, NJ). We refer especially to Figs. 3 and 4 in this paper. The authors associate the properties of the observed streamers with the viscosity of the perfluoro liquids used.

30. J. C. Adams, W. S. Brainerd, J. T. Martin, B. T. Smith, and J. L. Wagener, Fortran 90 Handbook (McGraw-Hill, New York, 1992). 